\title{
The N-terminal domain of thrombomodulin sequesters high-mobility group-B1 protein, a novel antiinflammatory mechanism
}

\author{
Kazuhiro Abeyama, ${ }^{1,2}$ David M. Stern, ${ }^{3}$ Yuji Ito, ${ }^{4}$ Ko-ichi Kawahara, ${ }^{1}$ Yasushi Yoshimoto, ${ }^{1}$ \\ Motoyuki Tanaka, ${ }^{1}$ Tomonori Uchimura, ${ }^{1}$ Nobuo Ida, ${ }^{5}$ Yoshiaki Yamazaki, ${ }^{5}$ Shingo Yamada, ${ }^{6}$ \\ Yasuhiko Yamamoto, ${ }^{7}$ Hiroshi Yamamoto, ${ }^{7}$ Satoshi lino, ${ }^{1}$ Noboru Taniguchi, ${ }^{1}$ and Ikuro Maruyama ${ }^{1}$ \\ 'Department of Laboratory and Molecular Medicine and 'Department of Preventive Medicine, Shin Nippon Biomedical Laboratories Inc. (SNBL), \\ Kagoshima University, Kagoshima, Japan. ${ }^{3}$ School of Medicine, Medical College of Georgia, Augusta, Georgia, USA. ${ }^{4}$ Department of Bioengineering, \\ Faculty of Engineering, Kagoshima University, Kagoshima, Japan. ${ }^{5}$ New Frontiers Research Laboratories Toray Industries, Kamakura, Japan. \\ ${ }^{6}$ Development Department, Shino-test Co., Sagamihara, Japan. ${ }^{7}$ Department of Biochemistry and Molecular Vascular Biology, \\ Kanazawa University Graduate School of Medical Science, Kanazawa, Japan.
}

\begin{abstract}
Thrombomodulin (TM) is an endothelial anticoagulant cofactor that promotes thrombin-mediated formation of activated protein $\mathrm{C}$ (APC). We have found that the $\mathrm{N}$-terminal lectin-like domain (D1) of TM has unique antiinflammatory properties. TM, via D1, binds high-mobility group-B1 DNA-binding protein (HMGB1), a factor closely associated with necrotic cell damage following its release from the nucleus, thereby preventing in vitro leukocyte activation, in vivo UV irradiation-induced cutaneous inflammation, and in vivo lipopolysaccharide-induced lethality. Our data also demonstrate antiinflammatory properties of a peptide spanning D1 of TM and suggest its therapeutic potential. These findings highlight a novel mechanism, i.e., sequestration of mediators, through which an endothelial cofactor, TM, suppresses inflammation quite distinctly from its anticoagulant cofactor activity, thereby preventing the interaction of these mediators with cell surface receptors on effector cells in the vasculature.
\end{abstract}

\section{Introduction}

Thrombomodulin (TM) was first identified as an endotheliumspecific membrane protein (1), and the TM-protein C (TM-PC) pathway is an essential anticoagulant-antithrombotic mechanism at least in part via inhibition of thrombin's procoagulant activity and limiting the generation of thrombin itself (1-6). The association of thrombin and TM on the endothelial surface also redirects the enzyme's substrate specificity toward activation of plasma PC (1-6), and activated protein C (APC) exerts additional anticoagulant effects by inactivating procoagulant cofactors Va and VIIIa $(2,3)$. Structurally, the extracellular portion of TM is composed of three domains: an N-terminal lectin-like domain (D1), followed by an EGF-like domain (D2) consisting of six EGF-like repeats, and an O-glycosylation-rich domain (D3) (4-6). D2 is critical for the protein's anticoagulant cofactor activities (i.e., inhibition of thrombin and promotion of APC formation) $(5,6)$.

Recent studies have shown that TM also plays an important role in attenuation of the inflammatory response in settings such as

Nonstandard abbreviations used: APC, activated protein C; D1, lectin-like domain; D2, EGF-like domain; D3, O-glycosylated domain; DCF-DA, 2' $7^{\prime}$-dichlorofluorescein diacetate; E456, fragment of TM spanning the last 3 EGF-like domains; HMGB1, high-mobility group-B1 DNA-binding protein; HPF, high-power field; HUVEC, human umbilical vein endothelial cell; MBP, maltose binding protein; P-D1, recombinant polypeptide corresponding to D1; P-D2+3, recombinant polypeptide corresponding to D2 and D3; PC, protein C; PMN, polymorphonuclear neutrophil; RAGE, receptor for advanced glycation endproducts; rhs-TM, recombinant human soluble TM; ROI, reactive oxygen intermediate; SEAP, secretory alkaline phosphatase; SPR, surface plasmon resonance; sRAGE, soluble RAGE; sRAGE-His, sRAGE with a polyhistidine tail; TM, thrombomodulin.

Conflict of interest: The authors have declared that no conflict of interest exists.

Citation for this article: J. Clin. Invest. 115:1267-1274 (2005).

doi:10.1172/JCI200522782. endotoxin-induced tissue damage, cytokine production, glomerulonephritis, and atherosclerosis (7-12). One mechanism for TM's antiinflammatory effects relates to properties of APC. For example, interaction of APC with the endothelial PC receptor triggers a cascade of antiinflammatory responses (13), which are quite distinct from the intrinsic anticoagulant properties of APC. The latter properties are likely to underlie, in large part, the success of the use of APC as a protective therapy in patients with sepsis (14). However, because of its strategic location on the vascular surface, we reasoned that TM itself might have the capacity to modulate inflammatory mechanisms. In this context, recent findings in mice expressing mutant TM variants and with TM peptide fragments in cell culture have pointed to a role for D1 of TM as a potential regulator of proinflammatory phenomena (15). Since the anticoagulant cofactor activity of TM is contained in D2, antiinflammatory properties of D1 have the potential to be distinct from APC formation.

High-mobility group-B1 DNA-binding protein (HMGB1) is a nuclear architectural chromatin-binding protein released by necrotic cells (16) that has recently been identified as a mediator of endotoxin-induced lethality (17) acting at least in part as a proinflammatory cytokine (18-21). Engagement of the receptor for advanced glycation endproducts (RAGE) $(22,23)$ by extracellular HMGB1 triggers activation of proinflammatory signaling pathways, such as those resulting in elaboration of reactive oxygen intermediates (ROIs) and NF-אB activation (23-25). Like TM, RAGE is also expressed on the endothelium (26), where it is optimally positioned to interact with HMGB1 released from cells damaged at the earliest stages of the host response. The starting point for our study was the hypothesis that TM might interact with a key inflammatory mediator, such as HMGB1, thereby preventing it from engaging cellular receptors (such as RAGE) and contributing to the inflammatory response. 
A
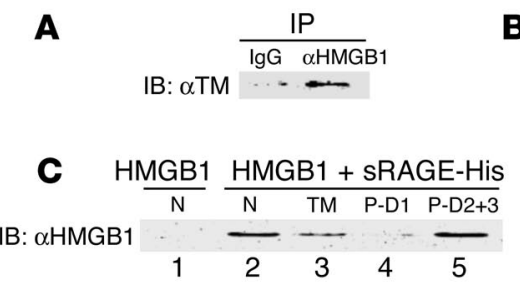

B

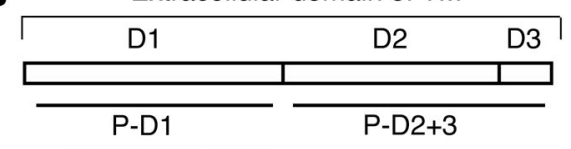

D1: lectin-like domain

D2: EGF-like domain

D3: O-glycosylated domain
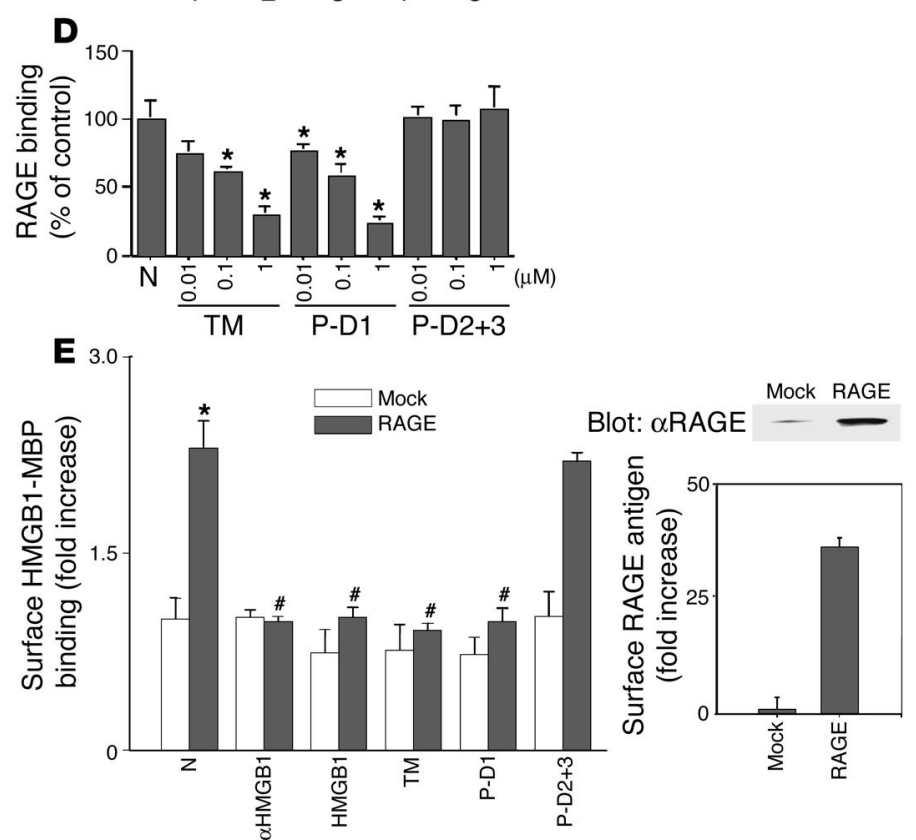

Figure 1

TM, via D1, binds to HMGB1. (A) For IP, rhs-TM (10 nM) and HMGB1 (10 nM) were mixed and incubated, followed by the addition of protein $A$ agarose beads conjugated to antiHMGB1 IgG ( $\alpha$ HMGB1) or nonimmune IgG (IgG). Immunoprecipitates were solubilized, and reduced SDS-PAGE (10\%) was followed by immunoblotting with anti-TM antibody ( $\alpha$ TM). (B) TM extracellular domains. (C) Competition between TM and sRAGE for binding to HMGB1. First, sRAGE-His (10 nM) was incubated with HMGB1 alone (10 nM; lane 2) or in the presence of rhs-TM (TM, $1 \mu \mathrm{M}$; lane 3), P-D1 (1 $\mu \mathrm{M}$; lane 4), or P-D2+3 (1 $\mu \mathrm{M}$; lane 5). Then, nickel resin beads were added, precipitates were solubilized, and SDS-PAGE (10\%) was followed by IB with $\alpha$ HMGB1 IgG. N, untreated controls. (D) Binding of sRAGE-His (10 $\mathrm{nM}$ ) to HMGB1 immobilized on plastic plates was studied with untreated controls, TM, P-D1, or P-D2+3. (E) Left panel: Binding of HMGB1-MBP (1 nM) to RAGE-transfected (RAGE) or mock-transfected (Mock) COS-7 cells ( $10^{4}$ cells/well) was assessed in the presence of untreated controls, $\alpha$ HMGB1, HMGB1, TM, P-D1, or P-D2+3 (100 nM each). Results shown are representative of 4 replicate wells. The right panel shows RAGE expression in RAGEtransfected versus mock-transfected COS-7 cells by IB (upper) and immunofluorescence on nonpermeabilized fixed cells (lower). ${ }^{*} P<0.05$, compared with the control group. ${ }^{\#} P<0.05$, compared with the paired, untreated controls.

Our results demonstrate that TM does bind HMGB1, thereby preventing its interaction with RAGE and suppressing induction of proinflammatory events, and that this interaction occurs at the level of D1 of TM. Furthermore, a peptide spanning D1 prevents UV irradiation-induced skin injury and lipopolysaccharide-induced (LPS-induced) lethality in mice. These data provide insight into a novel antiinflammatory mechanism at the level of the vessel wall, i.e., TM sequestration of HMGB1, and suggest possible therapeutic potential of D1 of TM.

\section{Results}

TM binds to HMGB1. One of the hallmarks of the host inflammatory/procoagulant response is its localization to sites of injury

in the vessel wall. Thus, antiinflammatory and anti-thrombotic cofactors would be likely to have essential roles in limiting host response cascades to a perturbed region of the vasculature, potentially through their ability to sequester inflammatory mediators. In this context, previous studies have shown that TM, an anticoagulant cofactor of the vessel wall, also has antiinflammatory properties quite distinct from its effect on the coagulation system (13-15). Those considerations led us to hypothesize that TM might bind to HMGB1, a potent proinflammatory mediator released by necrotic cells, thereby preventing dissemination of phlogogenic stimuli. Co-incubation of recombinant human soluble TM (rhs-TM) and HMGB1 was followed by immunoprecipitation with anti-HMGB1 IgG and immunoblotting of the immune precipitates with anti-TM IgG (Figure 1A). An immunoreactive TM band was observed when anti-HMGB1 IgG was used for immunoprecipitation, but not when it was substituted by nonimmune IgG (Figure 1A).

Binding studies using surface plasmon resonance (SPR), performed to directly assess the interaction of TM and immobilized HMGB1, demonstrated dose-dependent binding in the nanomolar range (not shown). In view of previous findings indicating antiinflammatory effects of the $\mathrm{N}$-terminal portion of TM (i.e., distinct from the anticoagulant domain of TM; ref. 15), we considered the lectin-like region as the possible site for HMGB1 interaction with TM (Figure 1B). Thus, we generated a recombinant polypeptide corresponding to D1 (P-D1) and another corresponding to D2 and D3 (P-D2+3). SPR binding studies with HMGB1 immobilized on sensor chips and P-D1 in the mobile phase demonstrated nanomolar binding comparable to that observed with full-length TM (not shown). In contrast, there was no observed binding between HMGB1 and P-D2+3 (not shown).

Effect of TM-HMGB1 interaction on binding of HMGB1 to RAGE. RAGE is a signal transduction receptor that mediates, at least in part, the interaction of HMGB1 with certain cell types leading to cellular activation (25). Thus, it was important to understand the effect of the binding of TM to HMGB1 on the interaction of HMGB1 with RAGE. For these studies, soluble RAGE (sRAGE) with a polyhistidine tail (sRAGE-His) was incubated with HMGB1 in the presence/absence of rhs-TM, and the mixture was precipitated by the addition of nickel resin beads to adsorb sRAGE-His (Figure 1C). sRage spans the extracellular ligand-binding domain of RAGE (26). In experiments in cellular systems, sRAGE serves as a decoy by binding ligands and preventing their interaction with cell surface receptor (26). Immunoprecipitation of mixtures of sRAGE-His and HMGB1 with nickel beads, followed by immunoblotting of immune precipitates with anti-HMGB1 IgG, showed an HMGB1-immunoreactive band (Figure 1C, lane 2). This result 
is consistent with previous data indicating that RAGE binds to HMGB1. When sRAGE and HMGB1 were incubated in the presence of TM, the same immunoprecipitation-immunoblotting procedure showed the intensity of the HMGB1 band was reduced, indicating that sRAGE and TM compete for binding to HMGB1 (Figure 1C, lane 3). As might be predicted from these results, using a competitive binding assay with immobilized sRAGE-His, the addition of rhs-TM decreased, in a dose-dependent manner, the binding of HMGB1 to RAGE (Figure 1D).

Given our SPR results analyzing the interaction of TM fragments with HMGB1 (see above), we used these portions of TM in immunoprecipitation studies. When the immunoprecipitationimmunoblotting protocol was applied to mixtures sRAGE-His and HMGB1 incubated in the presence of P-D1, intensity of the HMGB1immunoreactive band was decreased (Figure 1C, lane 4). In contrast, the P-D2+3 fragment of TM did not inhibit immunoprecipitationimmunoblotting of HMGB1-sRAGE complex (Figure 1C, lane 5). Consistent with those data, the addition of increasing amounts of P-D1 to the competitive binding assay with sRAGE-His and HMGB1 led to dose-dependent inhibition of the binding of HMGB1 to RAGE, whereas the addition of P-D2+3 was without effect (Figure 1D).

In order to assess whether TM would intercept the interaction of HMGB1 with RAGE in a cell-based system, we performed binding experiments using an HMGB1-maltose binding protein (HMGB1-MBP) fusion protein and COS-7 cells stably-transfected to overexpress RAGE. RAGE-transfected COS-7 cells displayed a substantial increase in cell surface RAGE antigen, based on immunofluorescence, and an increase in total RAGE antigen, based on immunoblotting, compared with mock-transfected controls (Figure 1E, right panel). Incubation of HMGB1-MBP with RAGE-transfected COS-7 cells resulted in increased association of HMGB1-MBP with the cell monolayer (based on an assay for MBP) compared with that of mock-transfected controls (Figure 1E, left panel). The latter signal was blocked in the presence of an excess of rhs-TM, HMGB1 (i.e., free HMGB1 not bound to MBP), the P-D1 fragment of TM, or antibody against HMGB1 (Figure 1E, left panel). In contrast, the P-D2+3 fragment was without effect.

Taken together, these data are consistent with the concept that the P-D1 portion of TM mediates the interaction with HMGB1 and that TM-HMGB1 binding prevents RAGE engagement by HMGB1. Thus, the binding sites on HMGB1 for RAGE and P-D1 appear to overlap. Furthermore, as the anticoagulant cofactor activity of TM resides in the P-D2+3 fragment, these results indicate that the ability of TM to bind HMGB1 is due to P-D1 and is distinct from the anticoagulant ability.

Effect of TM on HMGB1-mediated cellular activation in vitro. We performed further experiments with purified HMGB1 protein and human monocytic THP-1 cells to determine whether TM might modulate HMGB1's properties as a proinflammatory cytokine due at least in part to interaction with RAGE. Incubation of HMGB1 with THP-1 cells increased the expression of an

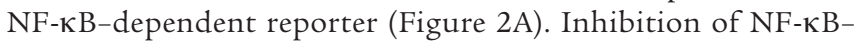
mediated transcription by the addition of sRAGE supported the view that HMGB1-RAGE interaction was involved in the activation of NF- $\kappa B$. Furthermore, in the presence of rhs-TM, HMGB1induced expression of the NF- $\mathrm{KB}$ reporter was suppressed to background levels (Figure 2A). Because ROIs have an important role in the signaling pathway leading to NF- $\mathrm{KB}$ activation in certain cases, we assessed whether HMGB1 would induce the generation of ROIs in a RAGE-dependent manner that could be inhib- ited by TM. Incubation of HMGB1 with THP-1 cells loaded with $2^{\prime}, 7^{\prime}$-dichlorofluorescein diacetate (DCF-DA) resulted in a prominent fluorescent signal (not shown). The addition of rhs-TM strongly suppressed the formation of the fluorescent adduct, as did the addition of P-D1. In contrast, P-D2+3 and a fragment of TM spanning the last 3 EGF-like domains (E456) (a truncated form of TM encompassing a minimal anticoagulant domain; ref. 6), TM fragments that do not include P-D1, were without effect (not shown). Thus, HMGB1-mediated activation of THP-1 cells, at the level of NF- $\mathrm{KB}$ activation and generation of ROIs, can be suppressed in the presence of the P-D1 portion of TM.

In order to extend these results using an established cell line, we isolated mononuclear phagocytes from human blood. Mononuclear phagocytes were preloaded with DCF-DA and were incubated with HMGB1. A fluorescent signal corresponding to oxidation of DCF-DA was observed in the presence of HMGB1. The addition of rhs-TM and P-D1, but not P-D2+3 or E456, resulted in attenuation of the fluorescent signal (Figure $2 \mathrm{~B}$ ). These data indicate that HMGB1-mediated activation of THP-1 cells and blood-derived mononuclear phagocytes can be prevented in the presence of P-D1 of TM, presumably by TM-mediated sequestration of HMGB1.

Role of HMGB1 in cutaneous inflammation. To further investigate the functional role of HMGB1 in inflammation and its potential regulation by TM, we used a cutaneous model of UV irradiationinduced inflammation. In preliminary experiments, we observed that a single dorsal exposure of the mouse ear skin to UV irradiation (wavelength, $254 \mathrm{~nm}$ ) caused marked inflammation that
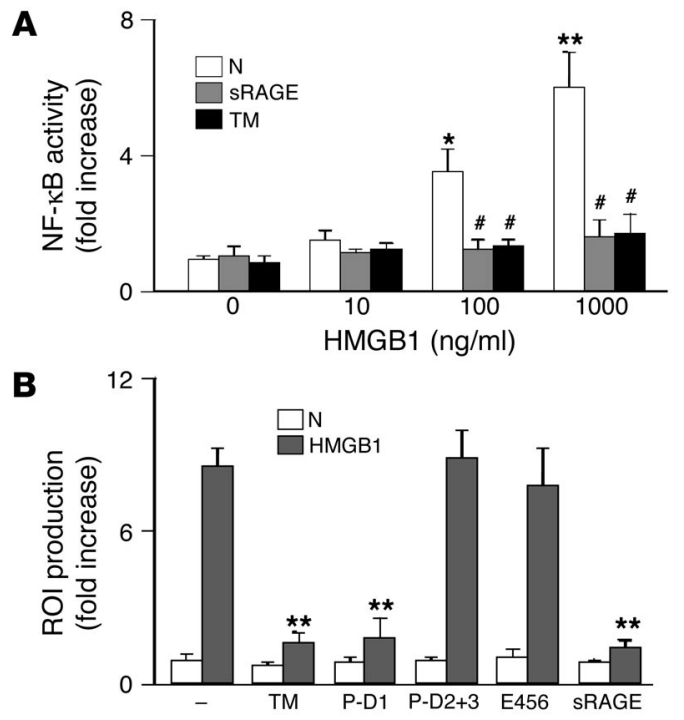

\section{Figure 2}

TM, via D1, prevents proinflammatory effects of HMGB1 in vitro. (A) Effect of HMGB1 on NF- $\mathrm{KB}-$ dependent gene transcription. THP-1 cells were transfected with an NF-kB promoter-reporter (SEAP) construct. Then, THP-1 cells $\left(10^{6} \mathrm{cells} / \mathrm{ml}\right)$ were incubated for 16 hours at $37^{\circ} \mathrm{C}$ with the indicated concentration of HMGB1, and reporter expression was determined. Recombinant sRAGE (10 nM) or rhs-TM (TM; 100 $\mathrm{nM}$ ) was added as indicated. (B) Human peripheral blood mononuclear phagocytes $\left(10^{6}\right.$ cells $\left./ \mathrm{ml}\right)$ were loaded with DCF-DA and were incubated for 1 hour at $37^{\circ} \mathrm{C}$ in medium alone (N) or with HMGB1 (10 nM) in medium. As indicated, rhs-TM, P-D1, P-D2+3, E456, or sRAGE was added (100 nM each). Then, the formation of DCF was determined. ${ }^{*} P<0.05$ and ${ }^{* *} P<0.01$, compared with untreated controls. ${ }^{*} P<0.05$, compared with the paired control group without sRAGE treatment. 



F

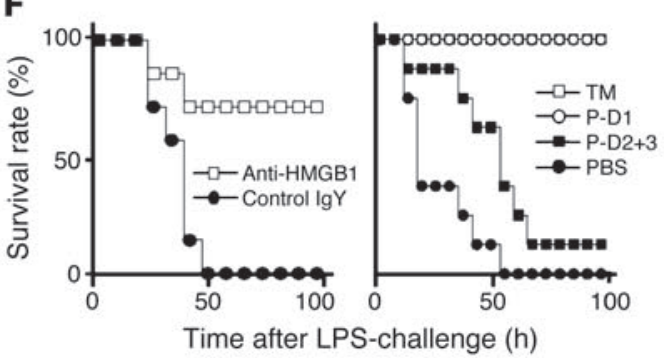

Figure 3

Central role of HMGB1 in UV irradiation-induced skin injury and the effect of TM and TM-derived peptides on HMGB1-mediated local and systemic inflammation. (A) In vivo effect of HMGB1 blockade on UV irradiationinduced cutaneous inflammation. The time course for ear swelling was measured after local subcutaneous administration of PBS or sRAGE (3 pmol; top); anti-HMGB1 IgY (Anti-HMGB1) or nonimmune IgY (Control IgY, 300 ng; middle); or rhs-TM (3 pmol) or PBS before exposure to UV irradiation (bottom). In each case, data shown are mean \pm SD $(n=8) .{ }^{*} P<0.05 ;{ }^{* *} P<0.01$. (B-E) As indicated, mice received PBS, rhs-TM, P-D1, P-D2+3 (each at $100 \mathrm{nmol} / \mathrm{kg}$; i.p.) or sRAGE (25 nmol/kg; i.p.) at 1 hour and 12 hours after exposure to UV irradiation ( $n=8$ per group). Three days after UV treatment, ear swelling (B), general histopathological features of the skin (C; H\&E staining; magnification, $\times 100)$, infiltrating leukocytes (D; cells/HPF), and local TNF- $\alpha$ accumulation $(E)$ were assessed. The mean $\pm S D$ of 6 determinations is shown (B, D, and E). ${ }^{*} P<0.05$ and ${ }^{* *} P<0.01$, compared with the UV-irradiated control group given PBS. $\# P<0.01$, compared with untreated controls. (F) Effect of TM and TM-derived peptides on systemic LPSinduced lethal challenge. Left panel: mice $(B A L B / c)$ received LPS $(10 \mathrm{mg} /$ $\mathrm{kg}$; i.p.) in the presence of anti-HMGB1 $\lg Y$ or nonimmune $\lg Y$ (3 i.p. doses of $2 \mathrm{mg} / \mathrm{kg} / \mathrm{mouse}$ of $\lg Y$ at 2, 12, and 24 hours after LPS). Right panel: mice were treated with LPS as described above and also received rhs-TM, P-D1, P-D2+3, or PBS (3 i.p. doses of 100 $\mathrm{nmol} / \mathrm{kg} /$ mouse for each peptide). could be monitored at the early stages by swelling of the ear (27). Inflammation induced by UV irradiation $\left(1,000 \mathrm{~J} / \mathrm{m}^{2}\right)$ developed over 3 days (Figure 3A), was sustained for up to 5 days, and displayed evidence of tissue/cell damage (not shown). Such UV irradiation-induced skin injury was associated with an increase in nuclear expression of HMGB1, as well as leakage of HMGB1 from the nucleus to extranuclear areas (not shown).

To probe the mechanisms underlying the host response to UV irradiation-induced cutaneous injury, we administered sRAGE. In the presence of sRAGE, blunting of UV irradiation-induced ear swelling was observed (Figure 3A, upper panel). These data suggested the possibility that HMGB1-RAGE interaction might contribute to the inflammatory response, leading us to test the effect of anti-HMGB1 IgY (Figure 3A, middle panel); ear swelling was reduced and the extent of reduction was similar to that observed after administration of sRAGE. These data indicate that ear swelling in the UV irradiation model is highly dependent on HMGB1.

Based on a role for HMGB1 in this model of cutaneous inflammation, we examined the effect of TM on the HMGB1-mediated cutaneous inflammation. The administration of rhs-TM demonstrated a protective effect on UV irradiation-induced cutaneous injury (Figure $3 \mathrm{~A}$, lower panel). Furthermore, the antiinflammatory effect of TM was similar in extent to what we observed previously with sRAGE and anti-HMGB1 IgY, consistent with a similar mode of action; i.e., prevention of the interaction of HMGB1 with cell surface acceptor sites such as RAGE. To further characterize the antiinflammatory properties of TM in this system, we performed studies with P-D1 and P-D2+3. In UV irradiation-induced skin inflammation, systemic administration of rhs-TM, P-D1, or sRAGE resulted in a significant blunting of the inflammatory response, based on ear swelling (Fig- 
ure 3B). In contrast, the effect of P-D2+3, although showing a trend toward decreased ear swelling, did not achieve statistical significance (P-D2+3 has antiinflammatory effects in vivo that probably reflect its ability to support thrombin-mediated activation of PC, whereas P-D1 is devoid of anticoagulant activity). Histological studies to evaluate characteristics of the inflammatory response (Figure 3C) and quantification of leukocyte infiltration (Figure 3D) and local TNF- $\alpha$ antigen accumulation (Figure $3 E$ ) in affected skin each showed significant reductions in animals treated with rhs-TM, P-D1, or sRAGE. These results demonstrate that HMGB1 is an important mediator of UV irradiation-induced skin injury and that TM, due to P-D1, attenuates the proinflammatory properties of HMGB1 in vivo.

Given the partially protective effect of treatment with either sRAGE or TM, we considered the possibility that a combination of both agents might have a more substantial effect on UV irradiation-induced cutaneous inflammation. For this purpose, animals were treated with TM or sRAGE alone or with sRAGE plus rhs-TM, P-D1, or P-D2+3. The effect on the inflammatory response was evaluated in terms of ear swelling (Figure $4 \mathrm{~A}$ ) and leukocyte infiltration of the skin (Figure 4B). No additive effect or synergy was observed; the protective effect of the administration of either sRAGE or TM was not bolstered by the presence of the other agent. These data are consistent with the idea that HMGB1 is the target for both agents.

In addition, it was important to determine the extent to which cell surface RAGE was the receptor sought out by HMGB1. This led us to repeat our studies of UV irradiation-induced dermal inflammation in homozygous RAGEnull mice. Studies performed in mice devoid of RAGE demonstrated prominent reduction in ear swelling (Figure 4A) and a partial decrease in leukocyte infiltration (Figure 4B) compared with that of strain-matched wild-type controls (RAGE-null and wild-type animals were on a C57BL/6 background). Administration of sRAGE, TM, or P-D1 to RAGE-null mice subjected to the UV irradiation-induced ear inflammation protocol displayed slightly increased protection with respect to ear swelling (Figure 4A) and a more prominent reduction in leukocyte infiltration (Figure 4B). These data indicate that cell surface RAGE, potentially on multiple cells in the dermis, is an important though not exclusive target of HMGB1 in UV irradiation-induced dermal inflammation.

The N-terminus-derived TM peptide has protective effects for LPS-induced lethality. Given recent data suggesting a link between HMGB1 released from injured tissue and endotoxininduced lethality in mice $(16,17)$, we tested whether rhs-TM and P-D1 might also have protective effects in this model (Figures

Figure 4
$3 \mathrm{~F}$ and $4 \mathrm{C})$. We used a dose of LPS (10 $\mathrm{mg} / \mathrm{kg})$ resulting in $100 \%$ lethality by 96 hours. Systemic (i.p.) treatment of animals with anti-HMGB1 IgY, administered 2, 12, and 24 hours after LPS, had a protective effect with respect to lethality at 4 days, whereas the same regimen using nonimmune IgY was without effect (Figures 3F and 4D). Similarly, i.p. administration of rhs-TM or P-D1, but not $\mathrm{P}-\mathrm{D} 2+3$ (in each case, according to the same schedule), had a complete protective effect comparable to that observed with antiHMGB1 IgY (Figure 3F and 4C).

Consistent with results of a previous study (28), RAGE-null mice exhibited a prominent decrease in LPS-induced mortality: 50\% lethali-
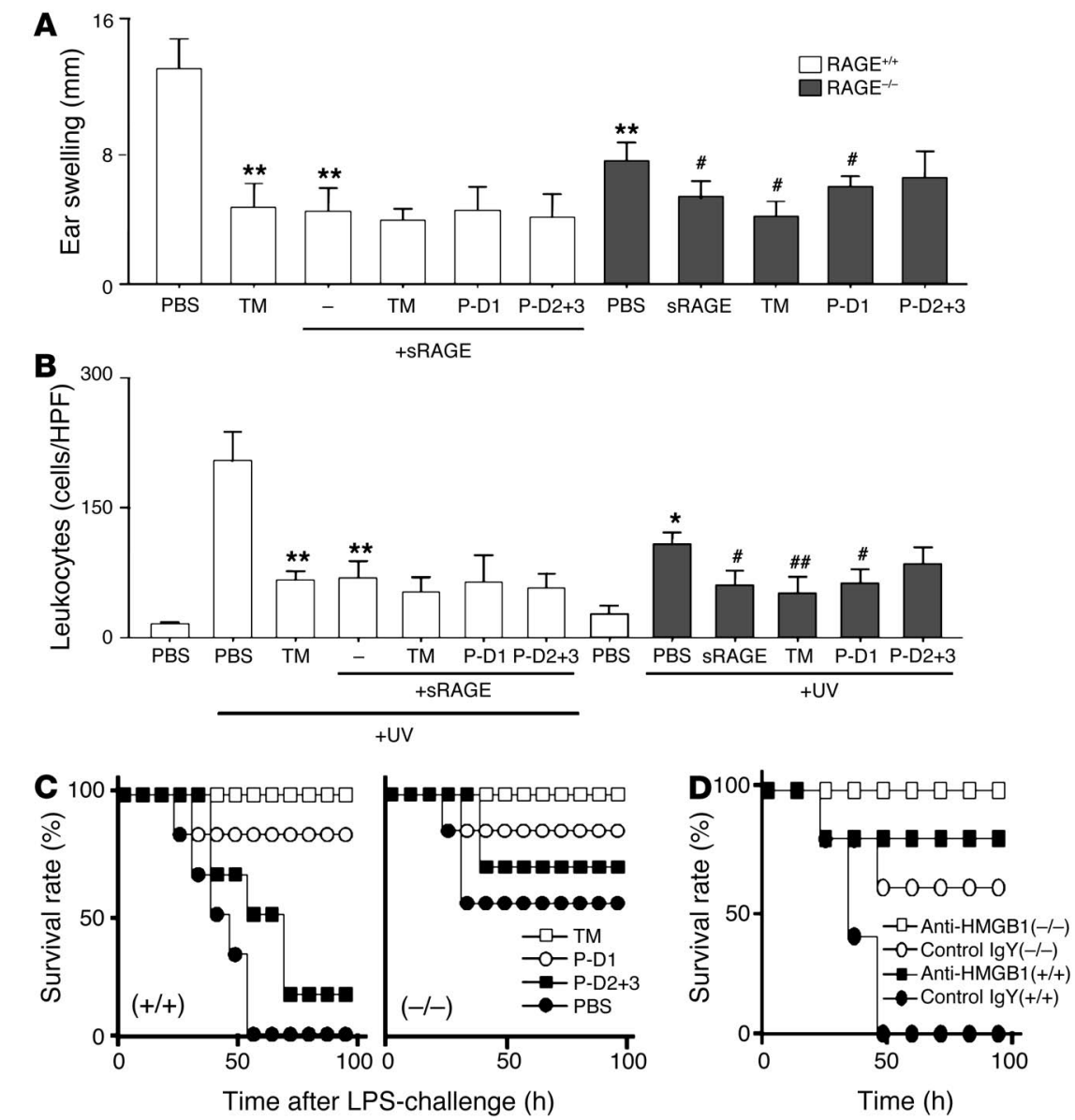

RAGE-dependent and -independent mechanism of HMGB1-mediated events and the inhibitory effects of TM and TM-derived peptides. (A and B) Skin inflammation was induced in the mouse ear using UV irradiation. C57/BL6 mice ( $R A G E^{+/+}$; white bars) received rhs-TM alone, sRAGE alone, or TM-derived peptides (each at $100 \mathrm{nmol} / \mathrm{kg}$; i.p.) combined with sRAGE ( $25 \mathrm{nmol} / \mathrm{kg}$; i.p.) at 1 hour and 12 hours after exposure to UV irradiation ( $n=8 \mathrm{per}$ group). RAGE-null mice (C57BL/6 strain; black bars) were UV irradiated and were treated with sRAGE $(25 \mathrm{nmol} / \mathrm{kg}$ ), rhs-TM, or TM-derived peptides (each at $100 \mathrm{nmol} / \mathrm{kg}$; i.p.). After 3 days, ear swelling $(\mathbf{A})$ and infiltrating leukocytes $(\mathbf{B})$ were assessed. ${ }^{*} P<0.05$ and ${ }^{* \star} P<0.01$, compared with UV-irradiated, PBS-treated controls. $\# P<0.05$ and $\# \# P<0.01$, compared with UV-irradiated RAGE-null mice. (C and D) Mice (wild-type C57BL/6 and RAGE-null on the C57BL/6 background) received LPS (10 mg/kg; i.p.) alone or in the presence of TM, P-D1, P-D2+3, and PBS. (C) Effect of rhs-TM or TM-derived peptides on LPSinduced lethality. Mice received 3 i.p. doses ( $100 \mathrm{nmol} / \mathrm{kg} / \mathrm{mouse})$ of anti-HMGB1 or control $\lg Y$ at 2, 12, and 24 hours after LPS challenge. (D) Mice were treated with LPS and also received either anti-HMGB1 $\mathrm{IgY}$ or nonimmune $\lg Y$ ( 3 i.p. doses of $2 \mathrm{mg} / \mathrm{kg} / \mathrm{mouse}$ of $\lg Y$ at 2, 12, and 24 hours after LPS). In C and D, (-/-) indicates that RAGE-null mice were used, and $(+/+)$ indicates that strain-matched controls were used. 
ty according to our protocol versus $100 \%$ for strain-matched C57BL/ 6 controls (Figure 4C). When RAGE-null mice were treated with antiHMGB1 IgY antibody, rhs-TM, or P-D1, mortality in response to LPS was almost completely eliminated (Figure 4, C and D). These data are consistent with the concept that RAGE-independent pathways also contribute to the lethality of LPS by a mechanism that appears to involve HMGB1 and appears to be prevented by P-D1 of TM.

\section{Discussion}

The initial notion of an endothelial cell surface that was inert with respect to procoagulant and proinflammatory events due to lack of expression of inducible cofactors, receptors, and mediators, such as tissue factor, E-selectin, and cytokines/chemokines, has undergone considerable revision (29). TM is particularly relevant in this context. As a high-affinity vascular receptor for thrombin, it binds circulating thrombin resulting in inhibition of the enzyme's procoagulant activity and promotion of PC activation (1-6), as well as preventing thrombin's interaction with protease-activated receptors (30). APC has direct anticoagulant properties at least in part in concert with protein S (31). In addition, this enzyme appears to have a spectrum of antiinflammatory properties $(8,14)$ that may be related to events mediated at the level of the endothelial PC receptor (13).

Another aspect of the biology of TM was suggested by infusion studies in which rhs-TM demonstrated protective properties in a range of models, including glomerulonephritis, sepsis, and ischemic settings (8-11). In these models, anticoagulation alone does not reproduce the effects of TM infusion $(32,33)$. Although the antiinflammatory properties of APC could and are likely to contribute, we suspected that TM itself might be involved. The first evidence supporting this view was obtained from the studies of Conway and colleagues (15) demonstrating that transgenic mice lacking D1 of TM displayed enhanced vulnerability to LPS challenge, with greater accumulation of polymorphonuclear neutrophils (PMNs) in their lungs and reduced survival. Furthermore, experiments in cell culture using recombinant D1 of TM showed suppression of PMN-EC adhesion, and decreased cytokine-induced activation of NF-кB and Erk1/2 (15). These data suggested that a polypeptide encompassing the lectin-like domain (called P-D1 in our studies) might have antiinflammatory properties in vivo.

The results of our studies demonstrate that TM binds HMGB1 via D1. These findings elucidate a novel and specific antiinflammatory property of TM that could be particularly relevant at the level of the vessel wall. Studies of HMGB1 have linked its release from cells to necrotic death $(16,17)$. Release of HMGB1 signals severe cellular damage and propagates an inflammatory response in neighboring cells as HMGB1 interacts with receptors such as RAGE (16-22). Binding of HMGB1 by TM provides a mechanism for damping such amplification of the inflammatory response. For example, in a severely injured vessel, one would expect loss of the integrity of cells bearing TM, including local release of TM $(32,34)$. Under these conditions, HMGB1 present in the extracellular environment would magnify the host response by binding to proinflammatory targets in the local milieu (16-20). However, once an adjacent portion of the vessel wall with intact endothelium is encountered, TM-bearing cells could sequester HMGB1, preventing the latter from reaching receptors with proinflammatory effects. Thus, the binding of TM and HMGB1 to each other could potentially "brake" the inflammatory process.

There are many questions raised by our results concerning mechanistic and practical considerations. It would be important to know if TM could bind HMGB1 and thrombin simultaneously, or if occupancy of TM by one would obviate binding of the other. The fate of the TM-HMGB1 complex would also be important to determine. For example, does the TM-HMGB1 complex dissociate, thereby "handing off" HMGB1 to other pathways involved in clearing HMGB1 from the circulation/cell surface? Also, the detailed pharmacokinetics of P-D1 and related molecules need to be worked out to determine if this approach is feasible from the standpoint of drug delivery.

Our findings have elucidated an unexpected antiinflammatory property of TM residing in D1, namely, binding of HMGB1. Although other protective properties may also be potential in this portion of TM, links between the protective effects of antiHMGB1 and the D1-derived peptide suggest that TM-HMGB1 interaction may be relevant to the inflammatory response in vivo. Our results move the field forward by demonstrating a novel antiinflammatory mechanism, sequestration of HMGB1 by TM, and suggesting a potential means for exploiting this observation in the development of a therapeutic agent based on D1.

\section{Methods}

Cells and cell culture. Human leukemia THP-1 cells were obtained from the American Type Culture Collection. Human peripheral blood mononuclear phagocytes were harvested by differential centrifugation and adherence (the mononuclear phagocyte population) from human blood (21). Human umbilical cord endothelial cells (HUVECs) were cultured from umbilical cords according to established methods (3). All procedures involving human materials were conducted under the auspices of a protocol approved by the institutional review board of Aiiku Hospital and Kagoshima University, Kagoshima, Japan. THP-1 cells and HUVECs were cultured in RPMI 1640 medium supplemented with $10 \%$ FCS, penicillin $(100 \mathrm{U} / \mathrm{ml})$, and streptomycin sulfate $(100 \mu \mathrm{g} / \mathrm{ml})$. The monkey kidneyderived cell line COS-7 was maintained in DMEM supplemented with the same additives described above. For the generation of RAGE-overexpressing cells, cDNA encoding full-length RAGE was cloned into the pCI-neo vector, and then the cDNA construct was transfected into COS-7 cells (35). Stable transfectants were selected in DMEM supplemented with G418 $(750 \mu \mathrm{g} / \mathrm{ml})$. RAGE expression in transfected COS-7 cells was characterized by immunoblotting (for total RAGE expression) and fluorescence immunostaining on nonpermeabilized cells (for cell surface RAGE expression) using polyclonal anti-RAGE IgG (35). For determining RAGE expression, the COS-7 monolayer was fixed with formaldehyde (4\%) and "reacted with" anti-RAGE IgG, and then the amount of bound IgG on cell surface was measured using FITC-conjugated second antibody.

Mice. Female BALB/C mice and C57BL/6 mice (8-10 weeks old; purchased from Japan SLC) were housed in the pathogen-free facility of the Animal Resource Center, Kagoshima University. RAGE-null mice, backcrossed into the C57BL/6 strain (F7), were provided by Hiroshi Yamamoto (Kanazawa University, Kanazawa, Japan). Experiments involving animals were approved by the Institutional Animal Care and Use Committee of Kagoshima University, Kagoshima, Japan, and were conducted according to NIH guidelines.

Anti-HMGB1 IgY neutralizing HMGB1. For the preparation of monospecific anti-HMGB1 antibody, IgY-class antibody from the egg yolk of HMGB1-immunized hens was isolated and purified according to the method of Hassl and Aspock (36).

Assays for TM-HMGB1 interaction. For immunoprecipitation, rhs-TM, prepared as described $(5,6,37)$, and purified human HMGB1 $(18,38)(10 \mathrm{nM}$, in each case) were incubated for 1 hour at $37^{\circ} \mathrm{C}$ in immunoprecipitation buffer (20 mM Tris-HCl, $150 \mathrm{mM} \mathrm{NaCl}, 1 \% \mathrm{NP}-40,1 \mathrm{mM}$ PMSF, and $0.1 \%$ $\mathrm{BSA}$; final volume, $1 \mathrm{ml}$ ). Then, protein A agarose beads conjugated with polyclonal anti-HMGB1 (approximately $10 \mu \mathrm{g}$ of $\mathrm{IgG}$ was adsorbed to $20 \mu \mathrm{l}$ 
of protein A agarose bead solution) were added to protein mixtures (200 $\mu \mathrm{l}$ each) and samples were incubated for an additional 1 hour. Protein A agarose was harvested by centrifugation and the immune precipitate was solubilized in reducing SDS-PAGE sample buffer ( $2 \%$ SDS; $50 \mu l)$. Samples (10 $\mu \mathrm{l} /$ lane) were subjected to reduced SDS-PAGE (10 \%) followed by immunoblotting with monoclonal anti-HMGB1 IgG (BD Biosciences - Pharmingen). Sites of binding of the primary antibody were visualized with second antibody conjugated to HRP. For determining the ability of TM to inhibit the binding of RAGE to HMGB1, the following pull-down assay was developed. The sRAGE-His was prepared as described previously $(23,39)$. Briefly, cDNA encoding the extracellular domain of RAGE was cloned into the $\mathrm{PQE}$ vector (Qiagen Inc.). With a nickel resin column, recombinant histidine-tagged sRAGE was purified from extract of Escherichia coli transformed with the vector. HMGB1 and sRAGE-His ( $10 \mathrm{nM}$ in each case) were incubated for 1 hour at $37^{\circ} \mathrm{C}$ in PBS (200 $\mu$ l per sample) containing $0.1 \%$ BSA. Nickel resin beads $(20 \mu \mathrm{l})$ were then added to precipitate sRAGE-His, the resin was harvested by centrifugation, and immune precipitates were solubilized in reducing SDSPAGE sample buffer ( $2 \%$ SDS; $50 \mu \mathrm{l})$. Samples $(10 \mu \mathrm{l} /$ lane $)$ were subjected to reduced SDS-PAGE (10\%) followed by immunoblotting with anti-HMGB1 $\operatorname{IgG}(0.2 \mu \mathrm{g} / \mathrm{ml})$. The effect of TM on sRAGE-HMGB1 interaction was studied by the addition of various TM-derived peptides at a concentration of $1 \mu \mathrm{M}$ for each: rhs-TM, P-D1, P-D2+3, and E456. The TM-derived peptides used in this study were prepared as described previously $(6,37)$.

A quantitative assay for assessing the ability of TM-derived peptides to block HMGB1-sRAGE interaction was developed as follows. A 96-well plate with aldehyde-activated amine-conjugated plastic (Sumitomo Inc.) was used to covalently link the C terminus of HMGB1. Then, sRAGE-His (10 nM) in PBS containing $0.1 \% \mathrm{BSA}$, in the presence/absence of the indicated concentrations (Figure 1C) of competitor TM-derived peptides, was added. Bound sRAGE was quantified using an antibody to the histidine tag of sRAGE, purchased from Qiagen Inc. For the cell-based HMGB1-RAGE binding assay, we cloned cDNA encoding HMGB1 into pMAL-c2X (New England Biolabs) to generate the recombinant HMGB1-MBP fusion protein. Briefly, RAGEtransfected cells (COS-7) or mock-transfected controls were incubated for 30 minutes at $37^{\circ} \mathrm{C}$ in DMEM with the HMGB1-MBP fusion protein under the indicated conditions (Figure 1E), followed by washing in PBS and fixation in formaldehyde (4\%). Bound HMGB1-MBP associated with the cell surface was quantified using an antibody to the MBP tag (Roche Diagnostics).

SPR analysis. Binding studies were performed by SPR using the BIAcore as described previously (40). In brief, HMGB1 was immobilized on the CM-5 sensor chips (BIAcore; Amersham Biosciences) using the $N$-ethyl- $N^{\prime}$-(dime thylaminopropyl)carbodiimide/ $N$-hydroxysuccinimide method according to the amine-coupling protocol recommended by the manufacturer. For association analysis, serial dilutions of TM-derived peptides or SRAGE in HBS buffer (10 mM HEPES buffer containing $0.15 \mathrm{M} \mathrm{NaCl}, 0.005 \%$ surfactant P20, and $3 \mathrm{mM}$ EDTA, $\mathrm{pH}$ 7.4) were injected onto the sensorchip at $20^{\circ} \mathrm{C}$ and at a flow rate of $20 \mu \mathrm{l} / \mathrm{min}$. The surface of the sensorchip was regenerated by elution with $0.2 \mathrm{M}$ glycine- $\mathrm{HCl}$ buffer, $\mathrm{pH}$ 2.7. Sensorgrams were analyzed to determine kinetic constants $\left(K_{\mathrm{a}}\right.$ and $\left.K_{\mathrm{d}}\right)$ by fitting of the data according to a 1:1 Langmuir dissociation/association model using the BIAevaluation 3.2 software. The results reported are approximate apparent $K_{\mathrm{d}}$ values reflecting a ratio of observed "on" and "off” rates.

Assessment of NF- $\mathrm{KB}$ activation and cytokines. NF- $\mathrm{KB}$-dependent transcription was monitored with a reporter gene assay (41). Briefly, cells were transfected with the NF- $\mathrm{kB}-$ specific reporter construct containing a $3 \times \mathrm{NF}-\kappa \mathrm{B}$ responsive element (heat-stable secretary alkaline phosphatase [SEAP]; Clontech Laboratory). After transfection and subsequent incubation under the indicated conditions (Figure 2A) for 16 hours, enzymatic activity of $\mathrm{SEAP}$ was measured. Levels of cytokines were assayed with a cytokine-specific ELISA kit for TNF- $\alpha$ (R\&D Systems Inc).
Monitoring the generation of ROIs. The generation of ROIs was assessed by incubation of cells for 30 minutes under the indicated conditions (Figure 2B) with DCF-DA $(5 \mu \mathrm{M})$, and the formation of the fluorescent adduct dichlorofluorescein (DCF) was measured by FACS analysis (Beckman-Coulter) or by using a fluorescent microplate reader (Fluoroscan; BD Biosciences - Pharmacia).

Cutaneous inflammation and LPS models. The dorsal surfaces of unrestrained BALB/c mice or RAGE-null mice were exposed to UV irradiation (wavelength, $254 \mathrm{~nm}$ ) at a dose of $1,000 \mathrm{~J} / \mathrm{m}^{2}$ using a UV Crosslinker lamp (Amersham Biosciences). Note that controls for experiments with RAGEnull mice (F7 in C57BL/6) used C57BL/6 animals. For assessment of the extent of skin inflammation, ear skin thickness was measured with a caliper-type engineer's micrometer (27). For histological analysis, ear samples were fixed in formalin and were stained with H\&E. Leukocyte infiltration was assessed semi-quantitatively by counting of the number of nuclei per high-power field (HPF; at least $20 \mathrm{HPFs}$ per sample were analyzed and 8 mice per experimental condition were studied). The extent of tissue damage was evaluated by TUNEL assay. Briefly, free 3'-OH DNA termini of DNA fragments were labeled with a digoxigenin-conjugated nucleotide. Digoxigenin-labeled DNA fragments were detected using a biotin-labeled antidigoxigenin. Certain ear specimens were homogenized and extracted in 10 mM HEPES/10 mM KCl buffer, pH 7.9, with 0.08\% NP-40, 0.1 mM EDTA, $1 \mathrm{mM}$ EGTA, $0.5 \mathrm{mM}$ DTT, and $0.5 \mathrm{mM}$ PMSF. Mixtures were centrifuged at $1,000 \mathrm{~g}$ for 20 minutes at $4{ }^{\circ} \mathrm{C}$. The resulting soluble fraction was considered to be derived from extranuclear protein (i.e., cytosolic material or that released from cells) and was assayed for HMGB1 and TNF- $\alpha$. Nuclear pellets were re-extracted in $20 \mathrm{mM}$ HEPES/0.4 M NaCl buffer, pH 7.9, $1 \mathrm{mM}$ EDTA, $1 \mathrm{mM}$ EGTA, $1 \mathrm{mM}$ DTT, and $1 \mathrm{mM}$ PMSF, and the supernatant, after centrifugation at $10,000 \mathrm{~g}$ for 60 minutes at $4^{\circ} \mathrm{C}$, was assessed for its HMGB1 content. Cellular localization of HMGB1 was evaluated immunohistochemically in inflamed skin tissue with monospecific anti-HMGB1 $\operatorname{IgG}(1 \mu \mathrm{g} / \mathrm{ml})$, prepared as indicated above. In studies assessing the effect of sRAGE and TM-derived peptides on inflammation, all materials were tested for LPS content with the Limulus amebocyte assay (LPS content was less than $0.25 \mathrm{pg}$ per $\mathrm{mg}$ of protein). TM-derived peptides (i.e., rhs-TM, P-D1, and P-D2+3) and sRAGE-His were prepared as described above.

For the systemic endotoxin challenge, LPS (from E. coli serotype O111:B4; purchased from Sigma-Aldrich) was administered i.p. Other agents were given as indicated (Figures $3 \mathrm{E}$ and 4, C and D).

Statistical analysis. Data were analyzed using Student's $t$ test, and $P$ values of less than 0.05 were considered significant.

\section{Acknowledgments}

We thank Asahi Chemical Co. for kindly providing rhs-TM and E456, and Satoshi Ogawa (Kanazawa University Graduate School of Medicine) and Takeo Fukuda (Kagoshima University) for helpful suggestions. This study was supported by research grants from the Ministry of Education, Culture, Sports, Science, and Technology of Japan: Grants-in-Aid 13470324 and 14657627 (to I. Maruyama), and 16659493 and 16390516 (to K. Abeyama).

Received for publication July 21, 2004, and accepted in revised form February 22, 2005.

Address correspondence to: Kazuhiro Abeyama and Ikuro Maruyama, Department of Preventive Medicine (SNBL), Kagoshima University, 8-35-1 Sakuragaoka, Kagoshima 890-8520, Japan. Phone: 81-99-275-5437; Fax: 81-99-275-2629; E-mail: k-abeyam@m3.kufm.kagoshima-u.ac.jp (Kazuhiro Abeyama). Phone: 81-99-275-5281; Fax: 81-99-275-2629; E-mail: rinken@m3. kufm.kagoshima-u.ac.jp (Ikuro Maruyama). 
1. Esmon, C.T., and Owen, G.W. 1981. Identification of an endothelial cell cofactor for thrombin-catalyzed activation of protein C. Proc. Natl. Acad. Sci. U. S. A. 78:249-252.

2. Esmon, C.T., Esmon, N.L., and Harris, K.W. 1982. Complex formation between thrombin and thrombomodulin inhibits both thrombin-catalyzed fibrin formation and factor $\mathrm{V}$ activation. J. Biol. Chem. 257:7944-7947.

3. Maruyama, I., Salem, H.H., and Majerus, P.W. 1984 Coagulation factor Va binds to human umbilical vein endothelial cells and accelerates protein $C$ activation. J. Clin. Invest. 74:224-230.

4. Esmon, C.T. 1989. The roles of protein C and thrombomodulin in the regulation of blood coagulation. J. Biol. Chem. 264:4743-4746.

5. Suzuki, K., et al. 1987. Structure and expression of human thrombomodulin, a thrombin receptor on endothelium acting as a cofactor for protein C activation. EMBO J. 6:1981-1997.

6. Zushi, M., et al. 1989. The last three consecutive epidermal growth factor-like structures of human thrombomodulin comprise the minimum functional domain for protein C-activating cofactor activity and anticoagulant activity. J. Biol. Chem. 254:10351-10353.

7. Weiler, H., et al. 2001. Characterization of a mouse model for thrombomodulin deficiency. Arterioscler. Thromb. Vasc. Biol. 21:1531-1537.

8. Uchiba, M., et al. 1996. Recombinant thrombomodulin prevents endotoxin-induced lung injury in rats by inhibiting leukocyte activation. Am.J. Pbysiol. 271:L470-L475.

9. Kaido, T., et al. 1999. Pretreatment with soluble thrombomodulin prevents intrasinusoidal coagulation and liver dysfunction following extensive hepatectomy in cirrhotic rats. Thromb. Haemost. 82:1302-1306.

10. Hasegawa, N., et al. 1996. The effects of recombinant human thrombomodulin on endotoxininduced multiple-system organ failure in rats. $A m$. J. Respir. Crit. Care Med. 153:1831-1837.

11. Ikeguchi, H., et al. 2002. Effects of human soluble thrombomodulin on experimental glomerulonephritis. Kidney Int. 61:490-501.

12. Waugh, J.M., et al. 2000. Thrombomodulin overexpression to limit neointima formation. Cir culation. 102:332-337.

13. Riewald, M., Petrovan, R.J., Donner, A., Mueller, B.M., and Ruf, W. 2002. Activation of endothelia cell protease activated receptor 1 by the protein $C$ pathway. Science. 296:1880-1882.

14. Bernard, G.R., et al. 2001. Recombinant human protein C Worldwide Evaluation in Severe Sepsis (PROWESS) study group. Efficacy and safety of recombinant human activated protein $\mathrm{C}$ for severe sepsis. N. Engl. J. Med. 344:699-709.

15. Conway, E.M., et al. 2002. The lectin-like domain of thrombomodulin confers protection from neutrophil-mediated tissue damage by suppressing adhesion molecule expression via nuclear factor $\mathrm{\kappa B}$ and mitogen-activated protein kinase pathways. J. Exp. Med. 196:565-577.

16. Scaffidi, P., Misteli, T., and Bianchi, M.E. 2002. Release of chromatin protein HMGB1 by necrotic cells triggers inflammation. Nature. 418:191-195.

17. Wang, H., et al. 1999. HMG-1 as a late mediator of endotoxin lethality in mice. Science. 285:248-251.

18. Taniguchi, N., et al. 2003. High mobility group box chromosomal protein 1 plays a role in the pathogenesis of rheumatoid arthritis as a novel cytokine. Arthritis Rheum. 48:971-981.

19. Pullerits, R., et al. 2003. High mobility group box chromosomal protein 1, a DNA binding cytokine, induces arthritis. Arthritis Rheum. 48:1693-1700.

20. Fiuza, C., et al. 2003. Inflammation-promoting activity of HMGB1 on human microvascular endothelial cells. Blood. 101:2652-2660.

21. Andersson, U., et al. 2000. High mobility group 1 protein (HMG-1) stimulates proinflammatory cytokine synthesis in human monocytes. J. Exp. Med. 192:565-570.

22. Taguchi, A., et al. 2000. Blockade of RAGE-amphoterin signalling suppresses tumour growth and metastases. Nature. 405:354-360.

23. Hori, O, et al. 1995. The receptor for advanced glycation end products (RAGE) is a cellular binding site for amphoterin. Mediation of neurite outgrowth and co-expression of rage and amphoterin in the developing nervous system. J. Biol. Chem. 270:25752-25761.

24. Shaw, S.S., et al. 2003. S100B-RAGE-mediated augmentation of angiotensin II-induced activation of JAK2 in vascular smooth muscle cells is dependent on PLD2. Diabetes. 52:2381-2388.

25. Huttunen, H.J., Fages, C., and Rauvala, H. 1999. Receptor for advanced glycation end products (RAGE)-mediated neurite outgrowth and activation of NF-KB require the cytoplasmic domain of the receptor but different downstream signaling pathways. J. Biol. Chem. 274:19919-19924.

26. Schmidt, A.M., Yan, S.D., Yan, S.F., and Stern, D.M. 2001. The multiligand receptor RAGE as a progression factor amplifying immune and inflammatory responses. J. Clin. Invest. 108:949-955. doi:10.1172/ JCI200114002.

27. Abeyama, K., et al. 2000. A role for NF-KB-dependent gene transactivation in sunburn. J. Clin. Invest. 105:1751-1579.

28. Liliensiek, B., et al. 2004. Receptor for advanced glycation end products (RAGE) regulates sepsis but not the adaptive immune response. J. Clin. Invest. 113:1641-1650. doi:10.1172/JCI200418704.

29. Cines, D.B., et al. 1998. Endothelial cells in physiology and in the pathophysiology of vascular disorders. Blood. 91:3527-3561.

30. Sarker, K.P., et al. 1999. Inhibition of thrombininduced neuronal cell death by recombinant thrombomodulin and E5510, a synthetic thrombin receptor signaling inhibitor. Thromb. Haemost. 82:1071-1077.

31. Dahlback, B. 1991. Protein S and C4b-binding protein: components involved in the regulation of the protein C anticoagulant system. Thromb. Haemost. 66:49-61

32. van der Poll, T., de Jonge, E., and Levi, M. 2001. Regulatory role of cytokines in disseminated intravascular coagulation. Semin. Thromb. Hemost. 27:639-651.

33. Riedemann, N.C., Guo, R.F., and Ward, P.A. 2003. The enigma of sepsis. J. Clin. Invest. 112:460-467. doi:10.1172/JCI200319523.

34. Abeyama, K., et al. 1995. Nonionic contrast agents produce thrombotic effect by inducing adhesion of leukocytes on human endothelium. Biochem. Biophys. Res. Commun. 212:776-783.

35. Yonekura, H., et al. 2003. Novel splice variants of the receptor for advanced glycation end-products expressed in human vascular endothelial cells and pericytes, and their putative roles in diabetesinduced vascular injury. Biochem. J. 370:1097-1109.

36. Hassl, A., and Aspock, H. 1988. Purification of egg yolk immunoglobulins. A two-step procedure using hydrophobic interaction chromatography and gel filtration. J. Immunol. Methods. 110:225-228.

37. Suzuki, K., et al. 1989. Domain composed of epidermal growth factor-like structures of human thrombomodulin is essential for thrombin binding and for protein C activation. J. Biol. Chem. 264:4872-4876

38. Yamada, S., Inoue, K., Yakabe, K., Imaizumi, H., and Maruyama, I. 2003. High mobility group protein 1 (HMGB1) quantified by ELISA with a monoclonal antibody that does not cross-react with HMGB2. Clin. Chem. 49:1535-1537.

39. Schmitt, J., Hess, H., and Stunnenberg, H.G. 1993. Affinity purification of histidine-tagged proteins. Mol. Biol. Rep. 18:223-230.

40. Hashiguchi, S., et al. 2003. Human Fc \& RI $\alpha$-specific human single-chain $\mathrm{Fv}(\mathrm{scFv})$ antibody with antagonistic activity toward IgE/Fc $\varepsilon$ RI $\alpha$-binding. J. Biochem. 133:43-49.

41. Abeyama, K., et al. 2003. Antibiotic cyclic AMP signaling by "primed" leukocytes confers anti-inflammatory cytoprotection. J. Leukoc. Biol. 74:908-915. 